\title{
Análise sensorial de pães de forma integrais enriquecidos com farinha de casca de jabuticaba
}

\section{Sensory analysis of whole-grain pan bread with the addition of jabuticaba peel flour}

\author{
Sumaya Patiara Lima Ferreira ${ }^{1}$; Fernanda Barbosa Borges Jardim²; Carolina Rodrigues da \\ Fonseca $^{3}$; Luciene Lacerda Costa ${ }^{4}$; Luciene Alves ${ }^{5}$
}

1Professora do Curso Técnico em Alimentos, Instituto Federal do Paraná, Jacarezinho, PR, Brasil. Orcid: http://orcid.org/0000-0002-8947-0708. E-mail: sumaya.ferreira@ifpr.edu.br

${ }^{2}$ Professora do PPG em Ciência e Tecnologia de Alimentos, Instituto Federal do Triângulo Mineiro, Uberaba, MG, Brasil. Orcid: http://orcid.org/0000-0002-4072-9889. E-mail: fernanda.jardim@iftm.edu.br

${ }^{3}$ Professora do PPG em Ciência e Tecnologia de Alimentos, Instituto Federal do Triângulo Mineiro, Uberaba, MG, Brasil. Orcid: https://orcid.org/0000-0003-2526-5913. E-mail: carolina@iftm.edu.br

${ }^{4}$ Técnica dos Laboratórios de Bromatologia e Análise Sensorial, Instituto Federal do Triângulo Mineiro, Uberaba, MG, Brasil. Orcid: http://orcid.org/0000-0002-9919-2369. E-mail: luciene@iftm.edu.br ${ }^{5}$ Professora do Curso de Graduação em Nutrição, Universidade Federal do Triângulo Mineiro, Uberaba, MG, Brasil. Orcid: http://orcid.org/0000-0003-4567-3359. E-mail: luciene.alves@uftm.edu.br

RESUMO: Os pães são muito usados para fins de enriquecimento nutricional e estão incluídos na dieta de todas as classes sociais. $O$ presente trabalho teve como objetivo analisar sensorialmente pães de forma integrais enriquecidos com farinha de casca de jabuticaba (FCJ) em diferentes proporções (controle $0 \%$, T1 2,5\%, T2 4,9\% e T3 7,2\%). O teste de aceitação sensorial foi conduzido com 50 julgadores não treinados, utilizando-se escala hedônica verbal estruturada de nove pontos (para os atributos sabor, cor, textura e aceitação global) e de três pontos para intenção de compra. Análises microbiológicas de coliformes a $45^{\circ} \mathrm{C}$ e Salmonella sp. foram realizadas nos pães, que apresentaram ausência destes microrganismos. A partir dos resultados obtidos do teste de aceitação, foram determinados os índices de aceitação. O pão controle e o pão com 2,5\% de FCJ apresentaram maior aceitação sensorial para os atributos impressão global e sabor com diferenças significativas em relação à T2 e T3, que não apresentaram diferenças entre si $(p<0,05)$. Não houve diferença significativa para os atributos cor, textura e aroma $(p<0,05)$. Comprovou-se que a adição de farinha de casca de jabuticaba afetou a decisão de compra do consumidor, visto que, de maneira geral, as médias de aceitação e os índices de aceitabilidade dos pães decresceram com o aumento da adição da FCJ, entretanto os pães com adição de 4,9 e 7,2\% de FCJ apresentaram resultados positivos, com notas próximas ou acima de 7,0, indicando potencial inserção de pães integrais com adição de farinha de casca de jabuticaba no mercado.

Palavras-chave: Aceitação; Jabuticaba; Panificação; Sensorial.

\begin{abstract}
Bread is widely used for the purpose of nutritional enrichment and is included in the diet of all social classes. The present work had the objective of sensorially analyzing whole wheat breads enriched with jabuticaba peel flour (JPF) in different proportions (control 0\%, T1 2.5\%, T2 4.9\% and T3 7.2\%). The sensory acceptance test was conducted with 50 untrained judges using the nine-point hedonic structured verbal scale (for the attributes of flavor, color, texture and overall acceptance) and a three-point scale for purchase intention. Microbiological analysis of coliforms at $45^{\circ} \mathrm{C}$ and Salmonella sp. were performed on the breads, which showed absence of these microorganisms. From the results obtained from the acceptance test, the acceptance indices were determined. The control bread and the bread with 2.5\% JPF presented greater sensory acceptance for the overall impression and flavor attributes with significant differences in relation to $T 2$ and $T 3$, which presented no differences between them $(p<0.05)$. There was no significant difference for the attributes of color, texture and aroma $(p<0.05)$. It was verified that the addition of jabuticaba peel flour affected the consumer purchase decision, since, in general, the averages of acceptance and the indices of acceptability declined with the increase of the JPF; however, the loaves with addition of $4.9 \%$ and $7.2 \%$ JPF presented positive results, with results close to 7.0, and thus indicating potential insertion of whole wheat bread with the addition of jabuticaba peel flour into the market.
\end{abstract}

Keywords: Acceptance; Jabuticaba; Baking; Sensory. 
Revista Brasileira de Ciência, Tecnologia e Inovação

\section{INTRODUÇÃO}

Muitos países têm adotado medidas para reduzir o consumo de alimentos processados na dieta da população, já que existe a suposição de que o consumo habitual desses produtos pode aumentar os fatores de risco do surgimento de doenças crônicas (FARDET; ROCK, 2019). Em virtude desta tendência, desencadeou-se, nos últimos anos, um aumento do interesse da indústria alimentícia pela pesquisa de novos compostos que possam ser utilizados para a elaboração de alimentos que promovam benefícios à saúde (DEL RÉ; JORGE, 2012; CONSTANTINO; LOPES, 2019).

Sabe-se que a farinha de casca da jabuticaba é rica em antocianinas e outros compostos fenólicos que, além de atuarem como antioxidantes, também possuem capacidade anti-inflamatória, atividade antimutagênica e anticancerígena, além de possuir um elevado teor de fibra dietética (LEITE-LEGATTI et al., 2012; SANTIAGO et al., 2018).

Os pães são muito usados para fins de enriquecimento nutricional, especialmente por serem uma das principais fontes calóricas da dieta, além de estarem incluídos na alimentação de todas as classes sociais (ENGINDENIZ; BOLATOVA, 2019). Portanto, são pertinentes investigações sobre ingredientes que possam melhorar características nutricionais de pães, de forma que mantenha sua produção economicamente viável (RIBEIRO; FERREIRA; FERREIRA, 2019). Desta forma, pães enriquecidos com farinha de casca de jabuticaba podem ser uma alternativa interessante para diversificar o setor, por agregar valor ao produto e contribuir positivamente para a manutenção da saúde (LENQUISTE et al., 2019).

A análise sensorial de produtos alimentares fornece indicações fundamentais para a produção e comercialização de produtos, no tocante às preferências e exigências do consumidor além de desempenhar papel de destaque no desenvolvimento de novos produtos (PALCZAK et al., 2019).

Portanto, este trabalho teve como objetivo analisar sensorialmente, a partir de testes de aceitação e intenção de compra, diferentes formulações de pães integrais adicionados de farinha de casca de jabuticaba.

\section{MATERIAL E MÉTODOS}

\section{Produção da farinha de casca de jabuticaba}

Frutos maduros de jabuticaba (Myrciaria jaboticaba), variedade Sabará, foram obtidos por doações da comunidade na cidade de Jacarezinho-PR, Brasil, no mês de setembro de 2016. Logo após o recebimento, a matéria-prima passou por seleção, limpeza e sanitização em solução de hipoclorito de sódio a $200 \mathrm{mg} \mathrm{L}^{-1}$ por 15 minutos. Em seguida, as jabuticabas foram despolpadas manualmente e as cascas foram dispostas em uma camada em assadeiras de alumínio. A secagem foi realizada em uma estufa com circulação de ar forçado na temperatura de $60^{\circ} \mathrm{C}$ por 12 horas (FERREIRA et al., 2012), e posteriormente trituradas com auxílio de um liquidificador doméstico. Após a obtenção, a farinha foi embalada em seladora a vácuo em sacos de nylon com polietileno de alta resistência e estocada em freezer a $-18^{\circ} \mathrm{C}$ até a utilização. 


\section{Produção dos pães de forma adicionados de farinha de casca de jabuticaba}

Os pães de forma foram produzidos utilizando uma formulação padrão (pão integral convencional) como tratamento controle e três tratamentos com adição de 2,5\% (T1), 4,9\% (T2) e 7,2\% (T3) de farinha de casca de jabuticaba (FCJ) (Tabela 1).

Tabela 1. Formulações de pães de forma integrais produzidos com adição de farinha de casca de jabuticaba (FCJ).

\begin{tabular}{lcccc}
\hline Ingredientes $^{*}$ & T0 & T1 & T2 & T3 \\
\hline Farinha de trigo especial & 20,5 & 20,0 & 19,5 & 19,1 \\
Farinha de trigo integral & 30,8 & 30,0 & 29,3 & 28,6 \\
Farinha de casca de jabuticaba & 0,0 & 2,5 & 4,9 & 7,2 \\
Água & 35,4 & 34,5 & 33,7 & 32,9 \\
Açúcar cristal & 6,2 & 6,0 & 5,9 & 5,7 \\
Gordura vegetal (80\% lipídeos) & 1,9 & 1,9 & 1,8 & 1,8 \\
Sal & 0,9 & 0,9 & 0,9 & 0,9 \\
Leite em pó integral & 1,5 & 1,5 & 1,5 & 1,4 \\
Fermento biológico seco instantâneo & 1,0 & 1,0 & 1,0 & 1,0 \\
Enzima alfa amilase & 0,5 & 0,5 & 0,5 & 0,5 \\
Anti- mofo propionato de cálcio & 0,1 & 0,1 & 0,1 & 0,1 \\
Glúten vital & 1,0 & 1,0 & 1,0 & 1,0 \\
\hline
\end{tabular}

${ }^{*}$ Porcentagens dos ingredientes das formulações de pães em base úmida. $\mathrm{T} 0=$ Pão de forma integral sem adição de farinha de casca de jabuticaba; $\mathrm{T} 1=$ Pão de forma integral com $2,5 \%$ de farinha de casca de jabuticaba; $\mathrm{T} 2=$ Pão de forma integral com $4,9 \%$ de farinha de casca de jabuticaba; $\mathrm{T} 3=$ Pão de forma integral com $7,2 \%$ de farinha de casca de jabuticaba. Fonte: SENAI, 2015.

Para preparação dos pães, foi efetuada a pesagem de todos os ingredientes da formulação em balança analítica e posterior acondicionamento em masseira automática Britânia modelo Multi Pane, primeiramente os ingredientes secos e depois os úmidos. $\mathrm{Na}$ masseira, houve as operações de mistura dos ingredientes, sova, descanso da massa, sova novamente, fermentação final e finalmente cocção dos pães, conforme instruções do Manual de Preparo de pães do equipamento Britânia. Após o cozimento, os pães foram desenformados e resfriados em temperatura ambiente, para serem fatiados manualmente em espessura média de $25 \mathrm{~mm}$ (Figura 1). As fatias foram embaladas em seladora sob vácuo em sacos de nylon com polietileno de alta resistência e estocadas em freezer a $18^{\circ} \mathrm{C}$ até a utilização.

\section{Determinação da qualidade microbiológica}

Os pães foram submetidos às análises microbiológicas exigidas pela Agência Nacional de Vigilância Sanitária - ANVISA, conforme diretrizes da Resolução - RDC no 12, de 02 de janeiro de 2001 (BRASIL, 2001) para esta categoria de produto alimentício, ou seja, Salmonella sp. e Coliformes a $45^{\circ} \mathrm{C}$, segundo metodologia de Silva et al. (2010). 
Figura 1. Pães de forma integrais com adição de 0 (T0), 2,5\% (T1), 4,9\% (T2) e 7,2\% (T3) de farinha de casca de jabuticaba em substituição parcial da farinha de trigo.

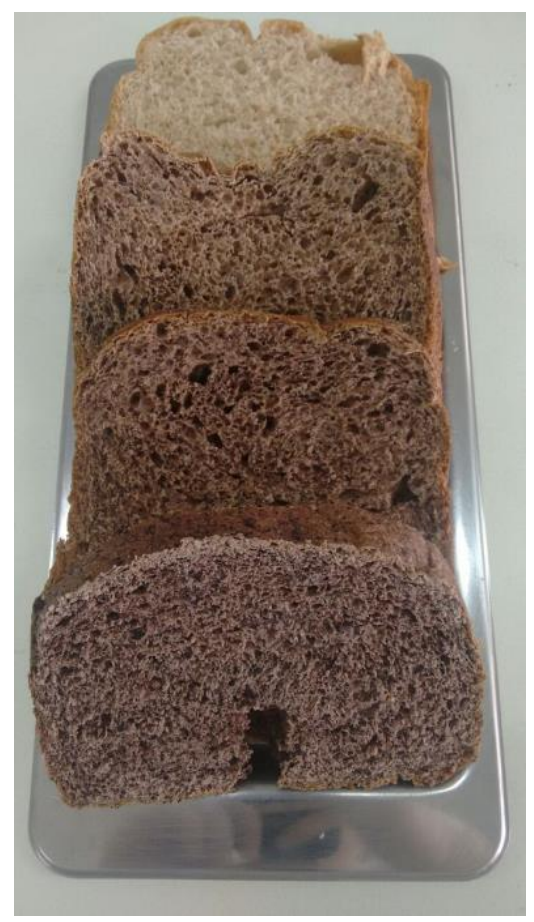

\section{Determinação da qualidade microbiológica}

Os pães foram submetidos às análises microbiológicas exigidas pela Agência Nacional de Vigilância Sanitária - ANVISA, conforme diretrizes da Resolução - RDC № 12, de 02 de janeiro de 2001 (BRASIL, 2001) para esta categoria de produto alimentício, ou seja, Salmonella sp. e Coliformes a $45^{\circ} \mathrm{C}$, segundo metodologia de Silva et al. (2010).

\section{Análise sensorial}

A análise sensorial dos pães de forma foi realizada com autorização do Comitê de Ética da Universidade Federal do Triângulo Mineiro - UFTM, sob no CAAE 43948015.7.0000.5154 no Laboratório de análise sensorial do Instituto Federal do Paraná - IFPR - campus Jacarezinho. O julgador, antes de realizar a análise sensorial, leu e assinou o Termo de Consentimento Livre, após esclarecimentos.

As formulações foram avaliadas sensorialmente por 50 julgadores, pelo teste de aceitação (MEILGAARD; CIVILLE; CARR, 1991). As amostras foram servidas na ordem balanceada de forma monádica.

No teste de aceitação, foram avaliados os atributos cor, aroma, sabor, textura e impressão global para cada formulação com a utilização de uma ficha contendo uma escala hedônica verbal estruturada de 9 pontos, onde $1=$ desgostei muitíssimo e 9 = gostei muitíssimo.

Para a intenção de compra, foi adotada a mesma ficha contendo uma escala verbal 


\section{RBC \\ RB \\ Revista Brasileira de Ciência, Tecnologia e Inovação}

estruturada de 3 pontos, variando de 1 = certamente não compraria e 3 = certamente compraria (MEILGAARD; CIVILLE; CARR, 1991; IAL, 2008).

Metodologia de análise dos resultados

Os resultados da análise sensorial e do teste de aceitação foram submetidos a delineamento em blocos completos com um controle e três tratamentos (adição de 2,5, 4,9 e 7,2\% de JPF), considerando 50 julgadores. Os efeitos dos tratamentos foram submetidos à análise de variância (ANOVA) e as médias classificadas pelo teste de Tukey a $5 \%$ de probabilidade para identificar as diferenças. Os resultados foram submetidos à análise estatística utilizando o software STATISTICA versão 13.0.

Para a intenção de compra, os resultados foram tabulados em porcentagem, variando de "eu certamente não compraria" até "eu certamente compraria o produto".

Para o cálculo de Índice de Aceitabilidade do produto, foi adotada a equação 1:

$$
\mathrm{I}(\%)=\mathrm{A} \times 100 / \mathrm{B}
$$

Em que IA é o índice de aceitabilidade, A é a nota média obtida para o produto e $B$ é nota máxima dada ao produto.

O IA com boa repercussão foi considerado $\geq 70 \%$ (DUTCOSKY, 2011).

\section{RESULTADOS E DISCUSSÃO}

\section{Resultados microbiológicos}

Além da qualidade nutricional, a qualidade microbiológica dos alimentos é essencial em termos de saúde e segurança do consumidor. Os pães desenvolvidos foram avaliados de acordo com os padrões microbiológicos previstos na RDC 12 de janeiro de 2001 (BRASIL, 2001), antes dos testes sensoriais (Tabela 2).

Tabela 2. Avaliação microbiológica dos pães de forma integrais com adição de diferentes proporções de farinha de casca de jabuticaba.

\begin{tabular}{|c|c|c|c|c|}
\hline \multirow[t]{2}{*}{ Análises microbiológicas } & \multicolumn{4}{|c|}{ Tratamentos } \\
\hline & T0 & T1 & $\mathrm{T} 2$ & T3 \\
\hline Coliformes a $45^{\circ} \mathrm{C} \mathrm{g}^{-1}\left(\mathrm{NMP} . \mathrm{g}^{-1}\right)^{*}$ & $<3,0$ & $<3,0$ & $<3,0$ & $<3,0$ \\
\hline Salmonella sp $25 \mathrm{~g}^{-1}$ (Ausência) & Aus** & Aus & Aus & Aus \\
\hline $\begin{array}{l}\text { "NMP.g }{ }^{-1} \text { : Número Mais Provável por g } \\
\text { T0= Pão de forma integral sem adição } \\
\text { T1 = Pão de forma integral com } 2,5 \% d e \\
\text { T2= Pão de forma integral com } 4,9 \% d e \\
\text { T3= Pão de forma integral com } 7,2 \% d e\end{array}$ & $\begin{array}{l}\text { us: Ause } \\
\text { e jabutic } \\
\text { jabutica } \\
\text { jabutica } \\
\text { jabutica }\end{array}$ & & & \\
\hline
\end{tabular}

A partir da análise dos resultados microbiológicos (Tabela 2), pôde-se afirmar que as quatro formulações estavam adequadas ao consumo no que diz respeito aos critérios microbiológicos, permitindo a utilização das amostras para os testes sensoriais. 
RBCTI

Resultados sensoriais

Os resultados das fichas dos testes sensoriais levaram a indicadores (Figura 2) de que a maior parte dos julgadores que avaliaram os pães eram mulheres (58\%), com idade abaixo dos 20 anos (74\%).

Figura 2. Perfil dos julgadores participantes nos testes sensoriais de aceitação e intenção de compra aplicados em pães de forma integrais com adição de diferentes proporções de farinha de casca de jabuticaba.
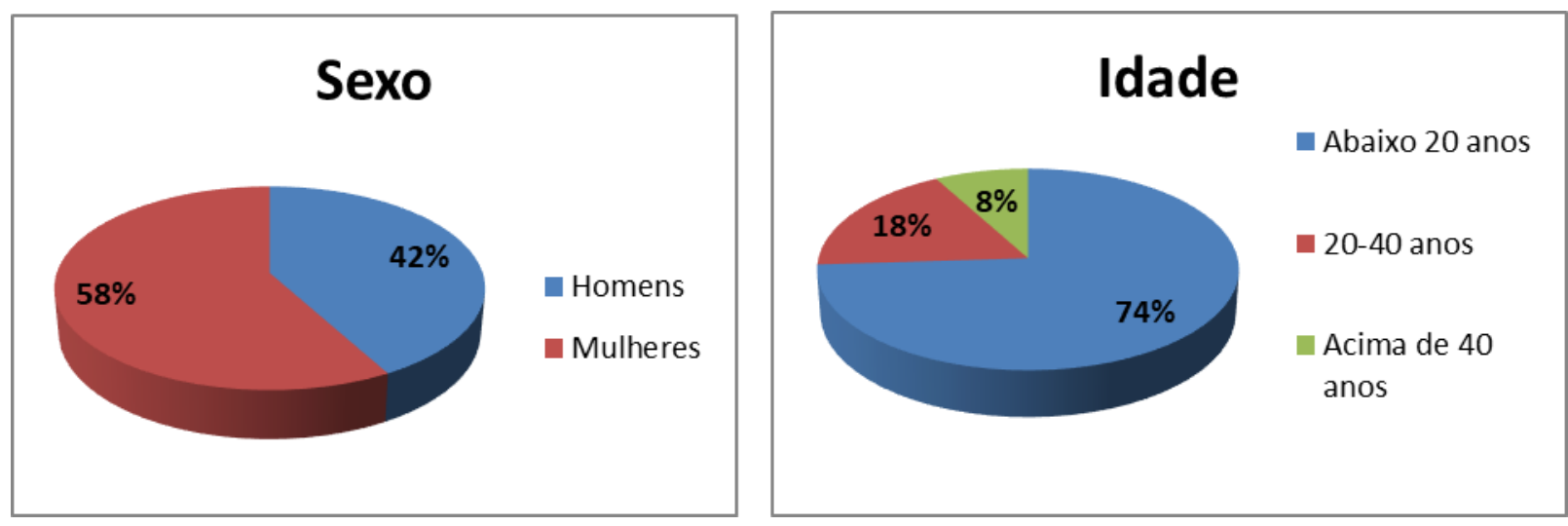

Os resultados do teste de aceitação das formulações de pães de forma estão apresentados na Tabela 3.

Tabela 3. Resultados da análise sensorial de aceitação dos pães de forma integrais com adição de diferentes proporções de farinha de casca de jabuticaba.

\begin{tabular}{|c|c|c|c|c|}
\hline \multirow[b]{2}{*}{ Atributos } & \multicolumn{4}{|c|}{ Tratamentos } \\
\hline & T0 & $\mathrm{T} 1$ & $\mathrm{~T} 2$ & T3 \\
\hline Cor & $7,58^{a} \pm 1,34$ & $7,18^{a} \pm 1,41$ & $6,92^{a} \pm 1,60$ & $7,14^{a} \pm 1,39$ \\
\hline Textura & $7,54^{\mathrm{a}} \pm 1,43$ & $7,18^{a} \pm 1,38$ & $7,38^{a} \pm 1,47$ & $6,96^{\mathrm{a}} \pm 1,81$ \\
\hline Sabor & $7,58^{a} \pm 1,51$ & $7,12^{a, b} \pm 1,41$ & $7,00^{\mathrm{a}, \mathrm{b}} \pm 1,71$ & $6,64^{b} \pm 1,63$ \\
\hline Aroma & $7,18^{a} \pm 1,69$ & $7,02^{a} \pm 1,49$ & $6,90^{a} \pm 1,68$ & $6,66^{a} \pm 2,02$ \\
\hline Impressão global & $7,76^{a} \pm 1,05$ & $7,37^{a} \pm 1,15$ & $6,43^{b} \pm 1,89$ & $6,47^{b} \pm 1,77$ \\
\hline $\begin{array}{l}\text { *Médias seguidas da mes } \\
\text { T0= Pão de forma integra } \\
\text { T1 = Pão de forma integra } \\
\text { T2= Pão de forma integra } \\
\text { T3= Pão de forma integra }\end{array}$ & $\begin{array}{l}\text { sma linha não } \\
\text { farinha de cas } \\
\text { arinha de casc } \\
\text { arinha de casc }\end{array}$ & $\begin{array}{l}\text { erem entre si p } \\
\text { de jabuticaba; } \\
\text { de jabuticaba; }\end{array}$ & Teste de Tuke & $(p<0,05)$ \\
\hline
\end{tabular}

Os pães T0, T1, T2 e T3 não diferiram estatisticamente entre si em relação aos atributos cor, textura e aroma.

Segundo Torbica et al. (2019), a cor é um dos atributos que mais causa impacto na avaliação do julgador, pois a partir da cor os consumidores avaliam a qualidade do produto como um todo, uma vez que geralmente associa-se a cor ao sabor e textura. Para o atributo referente a cor dos pães, foi possível observar que a adição da FCJ não afetou significativamente a coloração dos pães, conforme os julgadores. 
Revista Brasileira de Ciência, Tecnologia e Inovação

Os pães controle (T0) e T1 foram os mais aceitos em relação à impressão global, com diferenças significativas em relação a T2 e T3, que não apresentaram diferenças entre si. Os pães T0 e T1 receberam notas que se situaram entre "gostei moderadamente" e "gostei muito" e os pães T2 e T3 receberam notas que se situaram entre "gostei ligeiramente" e "gostei moderadamente" para o atributo impressão global (Tabela 3). Percebeu-se uma tendência de menor aceitação dos pães para o atributo impressão global, à medida que se aumentou as porcentagens de FCJ nas formulações.

Em relação ao atributo sabor, T1 e T2 não diferiram de T0 e T3, porém T0 e T3 diferiram entre si $(p<0,05)$. A mesma tendência foi observada para o atributo sabor, cujas formulações com maiores teores de FCJ obtiveram menores valores médios de aceitação.

Menores aceitações das formulações T2 e T3 eram esperadas, provavelmente devido à menor familiaridade dos provadores com um novo ingrediente (FCJ) em pães de forma.

Appelt et al. (2015) avaliaram três formulações de barras de cereais a base de farinha de okara com diferentes proporções de farinha de casca de jabuticaba e não identificaram diferenças significativas nos atributos aroma, cor e textura das formulações. Similarmente ao presente estudo para T0 e T1, as notas médias atribuídas pelos julgadores no teste de aceitação foram superiores a 7 em todos os atributos avaliados (cor, sabor, textura, aroma e impressão global) indicando boa aceitação pelos julgadores.

Ferreira et al. (2012) desenvolveram biscoitos tipo cookies adicionado da farinha de casca de jabuticaba e perceberam que todos os atributos avaliados no teste de aceitabilidade (cor, aparência, aroma, sabor, doçura, acidez textura) obtiveram os escores decrescentes a medida que aumentava o teor de farinha de casca de jabuticaba, corroborando com o presente estudo, para os atributos sabor e impressão global. Os autores concluíram que, de modo geral, os biscoitos sem adição e com adição de $5 \%$ foram os que apresentaram as maiores notas e índices de aceitabilidade pelos julgadores.

Eshak (2016) avaliou sensorialmente pães suplementados com 0\%, 5\% e 10\% de casca de banana e os resultados indicaram pequenas diferenças entre as notas de impressão global $(8,6,8,3$ e 8,2, respectivamente), decrescendo à medida que aumentava a concentração da casca de banana, assim como observado neste trabalho para os atributos sabor e impressão global.

Comumente é observado que as características sensoriais de pães são influenciadas pela adição de fibras de diferentes subprodutos da agroindústria (MARTINS; PINHO; FERREIRA, 2017). Neste trabalho, foi observado em outros estudos uma diminuição na aceitabilidade sensorial de pães com adição de diferentes produtos derivados de frutas, como casca de romã, pomelo, farinha de bagaço de uva, fibra de bagaço de abacaxi, casca de manga em pó, cascas e sementes de manga e fibra de limão (CHANG; LI; SHIAU, 2015; CHAREONTHAIKIJ; UAN-ON; PRINYAWIWATKUL, 2016; PATHAK et al., 2016; SULIEMAN et al., 2016; RESHMIA; SUDHAB; SHASHIREKH, 2017; ŠPORIN et al., 2018; IBRAHIM et al., 2018). Em muitos casos, a perda de aceitabilidade é justificada pela alteração da estrutura física, já que a adição de fibras prejudica a criação da estrutura aerada, característica principal do pão (QUILES et al., 2018; GÓMEZ; MARTINEZ, 2018).

Nesse estudo, todos os atributos dos pães apresentaram índices de aceitabilidade superiores à 70\% (Tabela 4), o que evidenciou uma aceitação sensorial satisfatória, pois Dutcosky (2011) cita que para que um produto seja aceito quanto a suas características 
sensoriais, é necessário que seu índice de aceitabilidade seja, no mínimo, de 70\%.

Entretanto, percebeu-se que à medida que se aumentou a proporção de FCJ, diminuíram os índices absolutos de aceitabilidade dos pães, como já relatado para os atributos impressão global e sabor.

Tabela 4. Índice de aceitação dos pães de forma integrais com adição de diferentes proporções de farinha de casca de jabuticaba.

\begin{tabular}{lcccc}
\multirow{2}{*}{\multicolumn{1}{c}{ Atributos }} & \multicolumn{4}{c}{ Tratamentos } \\
\cline { 2 - 5 } & $\mathrm{T} 0$ & $\mathrm{~T} 1$ & $\mathrm{~T} 2$ & $\mathrm{~T}$ \\
\hline Cor & 84,22 & 79,78 & 76,89 & 79,33 \\
Textura & 83,78 & 79,78 & 82,00 & 77,33 \\
Sabor & 84,22 & 79,11 & 77,78 & 73,78 \\
Aroma & 79,78 & 78,00 & 76,67 & 74,00 \\
Impressão global & 86,78 & 82,76 & 72,80 & 72,99 \\
\hline IC total (\%) & 83,54 & 79,42 & 77,54 & 75,72 \\
\hline
\end{tabular}

$\mathrm{T} 0=$ Pão de forma integral sem adição de farinha de casca de jabuticaba;

$\mathrm{T} 1=$ Pão de forma integral com $2,5 \%$ de farinha de casca de jabuticaba;

$\mathrm{T} 2=$ Pão de forma integral com $4,9 \%$ de farinha de casca de jabuticaba;

$\mathrm{T} 3=$ Pão de forma integral com $7,2 \%$ de farinha de casca de jabuticaba.

Khalil et al. (2017) avaliaram sensorialmente pães ázimo suplementados com farinha de banana verde (FBV) e obtiveram resultados similares ao observados neste trabalho. A amostra controle obteve maior índice de aceitabilidade total, de $95,75 \%$, com decréscimo dos índices, conforme maior proporção da farinha FBV: 93,50\% (10\% de FBV); $92,00 \%$ (20\% de FBV); $82,28 \%$ (30\% de FBV); $74,37 \%$ (40\% de FBV); $69,25 \%$ (50\% de FBV).

Semelhante a este estudo, Gül e Şen (2017) ao estudar os efeitos da substituição da farinha de trigo por farinha de semente de romã (FSR) em pães, na proporção de $0 ; 5 ; 7,5$ e 10\%, e Chareonthaikij, Uan-on e Prinyawiwatkul (2016) ao avaliar os efeitos da adição de fibra de bagaço de abacaxi (FBA) em pães na proporção de 0; 5 e 10\%, verificaram uma melhor avaliação sensorial nos pães adicionados de $5 \%$ de FSR e FBA, respectivamente.

$\mathrm{Na}$ Figura 3, são apresentados os resultados da intenção de compra dos julgadores em relação aos pães de forma integrais com diferentes proporções de FCJ.

As médias obtidas situaram-se, em geral, entre os escores 2 (tenho dúvidas se compraria) e 3 (certamente compraria) para todos os tratamentos. Os testes de intenção de compra foram compatíveis com os resultados do teste de aceitação, pois o T0 apresentou maiores notas para os atributos avaliados, seguido de T1 e T2 e T3. Portanto, comprovouse que a adição de farinha de casca de jabuticaba afetou a decisão de compra do consumidor, visto que, de maneira geral, as médias de aceitação decresceram com o aumento da adição da FCJ.

Constatou-se que a intenção de compra não teve relação direta com o índice de aceitação dos pães, principalmente para o T2, que apresentou uma intenção de compra de $50 \%$ para certamente compraria, enquanto para a impressão global obteve o menor valor $(6,43)$. A não concordância entre o índice de aceitação e a intenção de compra pode ocorrer pelo fato de ser um produto novo, que possui poucos similares no mercado. Outra justificativa pode ser o fato de as avaliações terem sido realizadas com julgadores não treinados, que representavam o público consumidor, com o qual desejava-se avaliar a 


\section{RBC RBC}

Revista Brasileira de Ciência, Tecnologia e Inovação

aceitabilidade dos pães de forma integrais com adição de FCJ.

Figura 3. Histograma de intenção de compra dos pães integrais com adição de diferentes proporções de farinha de casca de jabuticaba.

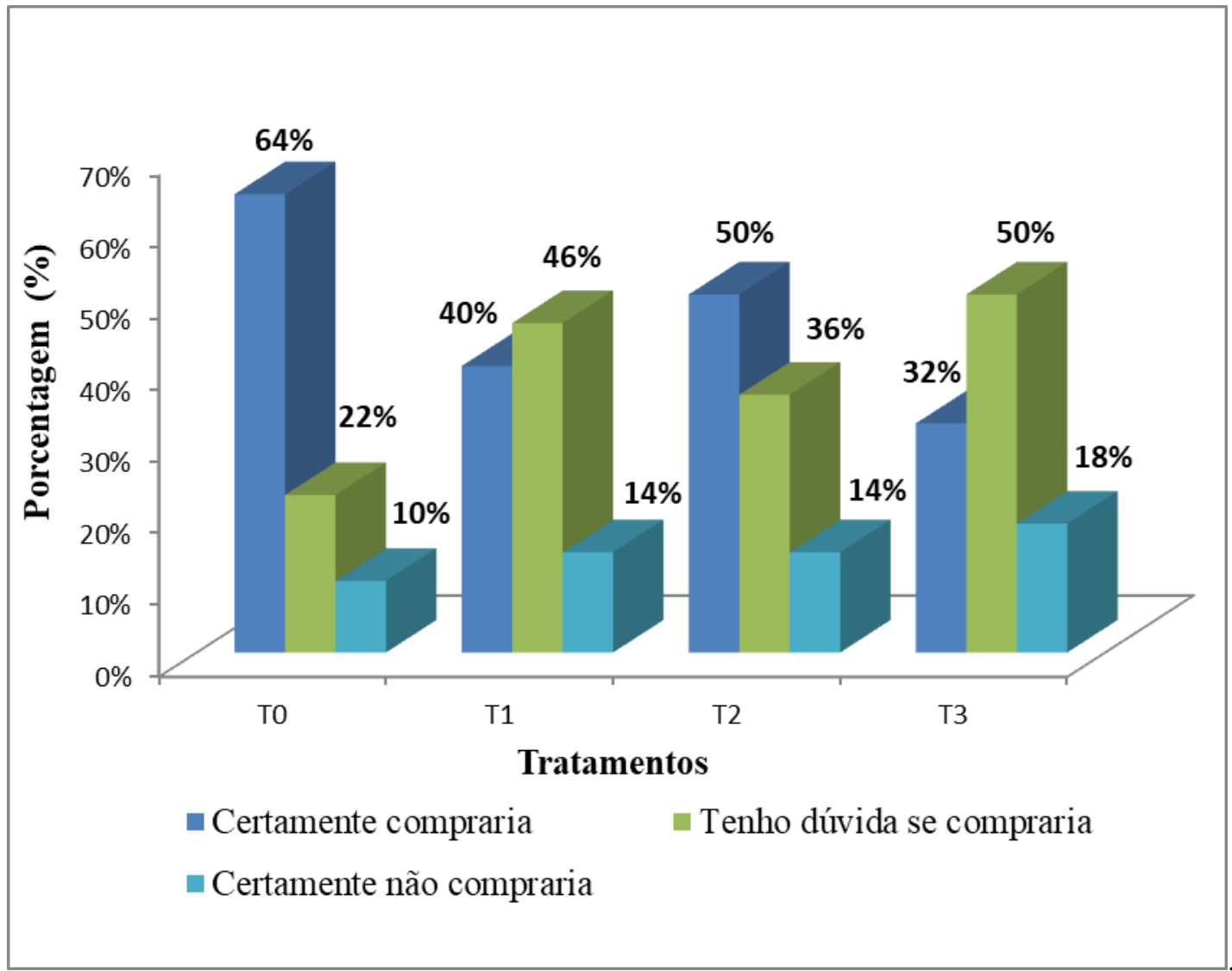

$\mathrm{T} 0=$ Pão de forma integral sem adição de farinha de casca de jabuticaba; $\mathrm{T} 1=$ Pão de forma integral com $2,5 \%$ de farinha de casca de jabuticaba; $\mathrm{T} 2=$ Pão de forma integral com $4,9 \%$ de farinha de casca de jabuticaba; $\mathrm{T} 3=$ Pão de forma integral com $7,2 \%$ de farinha de casca de jabuticaba.

\section{CONCLUSÕES}

De acordo com os resultados obtidos, foi possível concluir que os pães de forma integrais com adição de farinha de casca de jabuticaba atenderam aos padrões estabelecidos na legislação indicando, assim, a qualidade do produto panificável, estando apto para realização da análise sensorial.

O pão controle e o pão com 2,5\% de FCJ apresentaram maior aceitação sensorial em todos os atributos, entretanto os pães com adição de 4,9 e 7,2\% de FCJ apresentaram resultados positivos, com notas próximas ou acima de 7,0 , indicando potencial inserção de pães integrais com adição de farinha de casca de jabuticaba no mercado consumidor. 
RBCT]

\section{REFERÊNCIAS}

APPELT, P. et al. Development and characterization of cereal bars made with flour of jabuticaba peel and okara. Acta Scientiarum. Technology, v. 37, n. 1, p. 117, 2015. BRASIL. AGÊNCIA NACIONAL DE VIGILÂNCIA SANITÁRIA - ANVISA. Resolução RDC ANVISA no 12, de 02 de janeiro de 2001. Aprova o Regulamento Técnico sobre padrões microbiológicos para alimentos. Diário Oficial da União, Brasília, DF, Seção 1, p. 455310, 2001.

CHANG, R. C; LI, C. Y.; SHIAU, S. Y. Physico-chemical and sensory properties of bread enriched with lemon pomace fiber. Czech Journal of Food Sciences, v. 33, n. 2, p. 180185, 2015.

CHAREONTHAIKIJ, P.; UAN-ON, T.; PRINYAWIWATKUL, W. Effects of pineapple pomace fibre on physicochemical properties of composite flour and dough, and consumer acceptance of fibre-enriched wheat bread. International Journal of Food Science \& Technology, v. 51, n. 5, p. 1120-1129, 2016.

CONSTANTINO, J. S. F.; LOPES, D. L. Desenvolvimento de pão de forma contendo farinha mista de maracujá e jabuticaba. In: CARDOSO, N.A. et al. As ciências

biológicas e da saúde na contemporaneidade 2. Ponta Grossa: Atena Editora, 2019. p. 127-142.

DEL RÉ, P. V.; JORGE, N. Especiarias como antioxidantes naturais: aplicações em alimentos e implicação na saúde. Revista Brasileira de Plantas Medicinais, v. 14, n. 2, p. 389-399, 2012.

\section{DUTCOSKY, S. D. Análise sensorial de alimentos. Curitiba: DA Champagnat, 2011.}

ESHAK, N. S. Sensory evaluation and nutritional value of balady flat bread supplemented with banana peels as a natural source of dietary fiber. Annals of Agricultural Sciences, v. 61, n. 2 , p. 229-235, 2016.

ENGINDENIZ, S.; BOLATOVA, Z. A study on consumption of composite flour and bread in global perspective. British Food Journal, set. 2019. Disponível em:

<https://www.researchgate.net/publication/335918997_A_study_on_consumption_of_com posite_flour_and_bread_in_global_perspective>. Acesso em: 24 mar. 2021.

FARDET, A.; ROCK, E. Ultra-processed foods: A new holistic paradigm? Trends in Food Science \& Technology, v. 93, p. 174-184, 2019.

FERREIRA, A. E. et al. Produção, caracterização e utilização da farinha de casca de jabuticaba em biscoitos tipo cookie. Alimentos e Nutrição, v. 23, n. 4, p. 603-607, 2012.

GÜL, H.; ŞEN, H. Effects of pomegranate seed flour on dough rheology and bread quality. CyTA-Journal of Food, v. 15, n. 4, p. 622-628, 2017. 
RBCT]

GÓMEZ, M.; MARTINEZ, M. M. Fruit and vegetable by-products as novel ingredients to improve the nutritional quality of baked goods, Critical Reviews in Food Science and Nutrition, v. 58 n. 13, p. 2119-2135, 2018.

IAL. INSTITUTO ADOLFO LUTZ. Métodos físico-químicos para análise de alimentos. 4.ed. Brasília: Ministério da Saúde, 2008.

IBRAHIM, F. Y. et al. Effect of Substitution with Mango Peels and Seed Kernels as ByProducts on the Quality of Pan Bread and Cake. Journal of Food and Dairy Sciences, v. 9, n. 12, p. 439-445, 2018.

KHALIL, M. M. et al. Physiochemical and Sensory Evalution of some Bakery Products Supplemented with Unripe Banana Flour as a Source of Resistant Starch. Journal of Food and Dairy Sciences, v. 8, n. 10, p. 411-417, 2017.

LEITE-LEGATTI, A. V. et al. Jaboticaba peel: antioxidant compounds, antiproliferative and antimutagenic activities. Food Research international, v. 49, n. 1, p. 596-503, 2012.

LENQUISTE, S. A. et al. Jaboticaba peel powder and jaboticaba peel aqueous extract reduces obesity, insulin resistance and hepatic fat accumulation in rats. Food Research International, v. 120, p. 880-887, 2019.

MARTINS, Z. E.; PINHO, O.; FERREIRA, I. M. P. L. V. O. Fortification of Wheat Bread with Agroindustry By-Products: Statistical Methods for Sensory Preference Evaluation and Correlation with Color and Crumb Structure. Journal of Food Science, v. 82, n. 9, p. 2183-2191, 2017.

MEILGAARD, M.; CIVILLE, G. V.; CARR, B. T. Basic statistical methods. Sensory Evaluation Techniques, Boston: CRC Press, Inc., 1991. p. 237-273.

PALCZAK, J. et al. Sensory complexity and its influence on hedonic responses: A systematic review of applications in food and beverages, Food Quality and Preference, v. 71, p. 66-75, 2019.

PATHAK, D. et al. Characterization of physicochemical properties in whole wheat bread after incorporation of ripe mango peel. Journal of Food Measurement and Characterization, v. 10, n. 3, p. 554-561, 2016.

QUILES, A. et al. Fiber from fruit pomace: A review of applications in cereal-based products. Food Reviews International, v. 34, n. 2, p. 162-181, 2018.

RESHMI, M.L. SUDHA, M.N. SHASHIREKHA, Starch digestibility and predicted glycemic index in the bread fortified with pomelo (Citrus maxima) fruit segments, Food Chemistry, v. 237 , p. $957-965,2017$.

RIBEIRO, S. S.; FERREIRA J. C.; FERREIRA M. A. Análise do Processo de Comunicação dos Alimentos Funcionais: um Estudo das Embalagens de Pães de Fôrma. 
RBCTI

p. 259 -284. In: Tópicos em Ciências e Tecnologia de Alimentos: Resultados de Pesquisas Acadêmicas. São Paulo: Blucher, 2019. v. 4.

SANTIAGO, M. C. P. A. et al. Jabuticaba (Myrciaria jaboticaba (Vell.) O. Berg) peel powder produced by convective drying process: a rich anthocyanin product. Fruits. v. 73 n. 4, p. 201-208, 2018.

SENAI. Procedimento Operacional Padrão: Pão de Forma Integral. Uberaba: Serviço Nacional de Aprendizagem Industrial, 2015.

SILVA, N. et al. Manual de métodos de análise microbiológica de alimentos. 4. ed. São Paulo, SP: Varela, 2010.

ŠPORIN, M.; AVBELJ, M.; KOVAČ, B.; MOŽINA, S. S. Quality characteristics of wheat flour dough and bread containing grape pomace flour. Food Science and Technology International, v. 24, n. 3, p. 251-263, 2018.

SULIEMAN, A. M. E. et al. Influence of enrichment of wheat bread with pomegranate (Punica granatum L) peels by-products. International Journal of Food Science and Nutrition Engineering, v. 6, n. 1, p. 9-13, 2016.

TORBICA, A. et al. Sensory and physico-chemical properties of wholegrain wheat bread prepared with selected food by-products. LWT, v. 114, p. 1-8, 2019. 